\title{
Survival of Human Neurofibroma in Immunodeficient Mice and Initial Results of Therapy With Pirfenidone
}

\author{
Dusica Babovic-Vuksanovic, ${ }^{1 *}$ Lydia Petrovic, ${ }^{4}$ Bruce E. Knudsen, ${ }^{3}$ Timothy B. Plummer, ${ }^{3}$ \\ Joseph E. Parisi, ${ }^{4}$ Srdan Babovic, ${ }^{2}$ Jeffrey L. Platt ${ }^{2,3}$ \\ ${ }^{1}$ Department of Medical Genetics, Mayo Clinic, 200 First Street SW, Rochester, MN 55902, USA \\ ${ }^{2}$ Department of Surgery, Mayo Clinic, 200 First Street SW, Rochester, MN 55902, USA \\ ${ }^{3}$ Transplantation Biology Program, Mayo Clinic, 200 First Street SW, Rochester, MN 55902, USA \\ ${ }^{4}$ Department of Laboratory Medicine and Pathology, Mayo Clinic, 200 First Street SW, Rochester, MN 55902, USA
}

Received 27 September 2003; revised 16 December 2003; accepted 22 December 2003

\begin{abstract}
Neurofibromatosis type I is a common tumor predisposing disease in humans. Surgical therapy can be applied only in selected patients with resectable masses. Hence, development of new therapies for this disease is urgent. We used human neurofibroma implants in mice with severe combined immunodeficiency (SCID) as a model to test the toxicity and potential efficacy of pirfenidone, a new therapeutic agent. Two hundred twelve human neurofibromas were transplanted into various locations in 59 experimental animals, and 30 mice with implants received oral pirfenidone for up to six weeks. Survival of neurofibromas in animals treated with pirfenidone was lower than in the control group $(P=.02)$. Tumors did not change histologic appearance or vascularization in response to pirfenidone. Treatment with pirfenidone, a new antifibrotic agent, inhibits survival of some tumors without causing toxicity in animals.
\end{abstract}

\section{INTRODUCTION}

Neurofibromatosis type I (NF1) is an autosomal dominant condition affecting about 1 in 3000 individuals. The disorder shows extraordinary variability in clinical presentation but most frequently causes benign neural tumors, neurofibromas, and abnormal pigmentation in form of cafe-au-lait macules, intertriginous freckling of skin, and Lisch nodules of the iris. The diagnosis of NF1 is based on clinical criteria, as defined at an NIH Consensus Development Conference [1]. Individuals with NF1 are at risk for optic nerve gliomas, nerve root and plexiform neurofibromas, schwannomas, spinal cord tumors, benign and malignant peripheral nerve sheath tumors, and pheochromocytomas [2,3]. Plexiform neurofibromas tend to infiltrate the surrounding tissues causing disfigurement and compromise of nearby vital structures by compression. These tumors may be multiple and may show rapid growth throughout life, but the periods of greatest risk are puberty and childbearing age. Surgical removal of peripheral nerve tumors still remains the only therapeutic option; however, in many patients, tumors cannot be completely resected, and there is a high rate of regrowth (about 50\%), especially in young children after resection of tumors of the head/neck/face [4]. Therefore, new approaches to therapy that would arrest progressive course of NF1 are urgently needed [5]. One hurdle to developing new therapies for NF1 has been the lack of a suitable animal model. Attempts of implantation of human neurofibroma in experimental animals were made before $[6,7,8]$, but not in the subcutaneous location of a mouse ear, which has an advantage of easy accessibility of the tumor for monitoring the survival, growth, or changes in vascularization. We have developed a model in which fragments of human neurofibroma are implanted in various locations in immunodeficient mice and used the model for initial testing of an agent that might be useful in therapy of neurofibromatosis.

The agent we explored was pirfenidone, 5-methyl-1phenyl-2-(1H)-pyridone, a novel antifibrotic drug that has been shown to inhibit fibroblast growth and collagen synthesis. Pirfenidone modulates the action of such cytokines, as platelet-derived growth factor, fibroblast growth factor, epidermal growth factor, intercellular adhesion molecule-1 [9], and transforming growth factor beta-1 [10]. Inhibition of these cytokines decreases proliferation and collagen matrix synthesis by human fibroblasts. Antifibrotic effects of pirfenidone have been reported in animals, suggesting therapeutic potential in many fibrosing conditions $[11,12]$. The histopathology of neurofibromas is characterized by slender spindle cells with an abundant extracellular matrix of dense, wavy collagen fibers and extracellular mucoid material. Up to 70 percent of the tumor dry weight is collagen $[13,14]$. Although the understanding of molecular events that lead to the development of neurofibromas in NF1 is still incomplete, comparison of the effects of pirfenidone with the observed alterations in NF1 led to our hypothesis that 
pirfenidone might exert a therapeutic effect in NF1, by targeting fibrotic component of these tumors. We tested the toxicity of pirfenidone and evaluated efficacy of short course of therapy as a prelude to clinical trials.

\section{MATERIALS AND METHODS}

\section{Animals}

Mice with severe combined immunodeficiency (SCID) were used as recipients of human neurofibroma xenotransplants. SCID mice have a deficiency in the DNA-dependent protein kinase (DNA-PK), which results in inefficient recombination of DNA ends generated during the course of antigen receptor recombination [15]. This defect blocks lymphopoiesis at early stages of $\mathrm{T}$ - and B-cell development and prevents the mounting of immune responses against the grafts.

Two hundred twelve human neurofibromas were transplanted into various locations in 59 experimental animals, 30 in the epineurium of the sciatic nerve, and 182 under the skin of the neck, back, and skin of the ear. Thirty mice with implants received oral pirfenidone for up to six weeks, and 29 animals were monitored for survival of tumors without therapy.

\section{Human neurofibromas}

Human neurofibromas were obtained from four patients with neurofibromatosis who underwent removal of plexiform neurofibroma due to pain, compression, or other clinical indications. One tumor was described as a nodular plexiform neurofibroma, while the other 3 had appearance of diffuse plexiform neurofibroma. Tumor tissue that would normally be discarded was used for these experiments. Each tumor was initially evaluated by frozen section histology, and if confirmed to be a neurofibroma, tumor tissue was prepared for implantation. The size of the tumors ranged from $2 \mathrm{~cm}$ to $4 \mathrm{~cm}$ at the greatest diameter. The tissue was placed into sterile culture medium with an antibiotic and cut into $2 \times 2 \times 2 \mathrm{~mm}(1-2 \mathrm{mil}-$ ligrams) pieces. Two hundred and twelve tumor fragments were obtained by cutting neurofibromas originating from four different patients. Tumors were weighed and measured by a caliper (two largest perpendicular diameters) before implantation. Part of the tumor was evaluated by histology as described below.

\section{Surgical procedure}

Surgical procedures were conducted under sterile conditions. Each mouse was anesthetized using 5\% halothane inhalation followed by $1 \%-2 \%$ halothane for maintenance. Tumor fragments were implanted subcutaneously in the area of back, neck, and on the posterior surface of the ear. Prior to incision, the skin was thoroughly cleaned with $70 \%$ ethanol and Betadine solution. Incisions were performed longitudinally oriented on the skin of designated areas. The implantation pockets were dissected with microscissors. Care was taken to minimize mechanical trauma to the graft. After implantation of the tumor grafts, skin was closed with resorbable (Chromic catgut 5/0) suture. We used method previously described by Lee et al for implantation of neurofibroma fragment into the epineurium of the sciatic nerve [16]. Tumor implants were secured with a Prolene $8 / 0$ surgical suture.

\section{Pirfenidone therapy}

Tumors were implanted in four different occasions, depending on availability of the human donor. After the implantation of xenografts from each donor, animals were randomized into two groups. Thirty SCID mice with implanted tumor fragments in various body locations were treated with oral pirfenidone (at a dose of $500 \mathrm{mg} / \mathrm{kg}$ per day). Therapy was initiated the second day after tumor implantation. The remaining 29 untreated mice with implants from the same tumors were used as controls.

\section{Assessment of tumor growth}

The animals with tumor implants in the epineurium and under the skin of neck and back were followed for up to six weeks. The survival and size of tumor implants in subcutaneous locations were assessed by external palpation through the skin of the neck and back and measurement of the perpendicular diameters prior to tumor removal.

Animals were sacrificed 2, 3, 4, 5, and 6 weeks after tumor implantation. Neurofibroma implants were recovered, weighed, and measured for the largest surface area. Tumor tissue was prepared for routine histology and immunostaining. The authors were masked to a treatment status of animals at the time of tumor measurement to minimize bias.

Two animals with tumor implants under the skin of ear were followed for twelve months without therapy. Tumor appearance and size of tumors were assessed by inspection and direct outside measurement of the two largest perpendicular diameters and calculation of area, using a caliper with $0.025 \mathrm{~mm}$ accuracy (Skilltech, Hempe Manufacturing Co, Inc, New Berlin, Wis) (Figure 1a). Measurements were performed two weeks after implantation and then monthly thereafter.

\section{Histology and immunohistochemistry}

Tumor specimens were fixed in $10 \%$ neutral buffered formalin, $\mathrm{pH}=7.2$, for up to 18 hours at room temperature (RT), then embedded in paraffin using a tissue processor (model PTP 1530, Ventana Medical Systems, Tucson, Ariz). Sections prepared from these blocks were placed on positively charged microscope slides (Superfrost Plus, Fisher Scientific, Pittsburgh, Pa). The sections were then deparaffinized with xylene and rehydrated using graded ethanol solutions. For immunohistochemical methods, endogenous peroxidase was quenched by incubation of the sections with $1.5 \% \mathrm{H}_{2} \mathrm{O}_{2}$ in $50 \%$ methanol. 


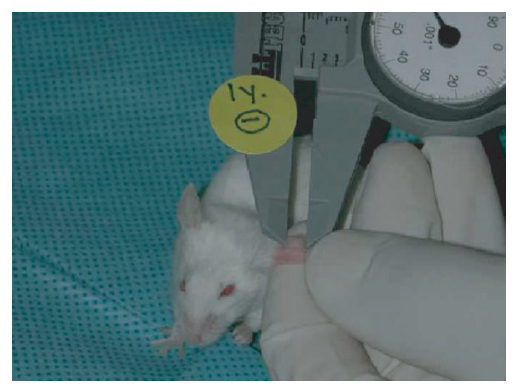

(a)

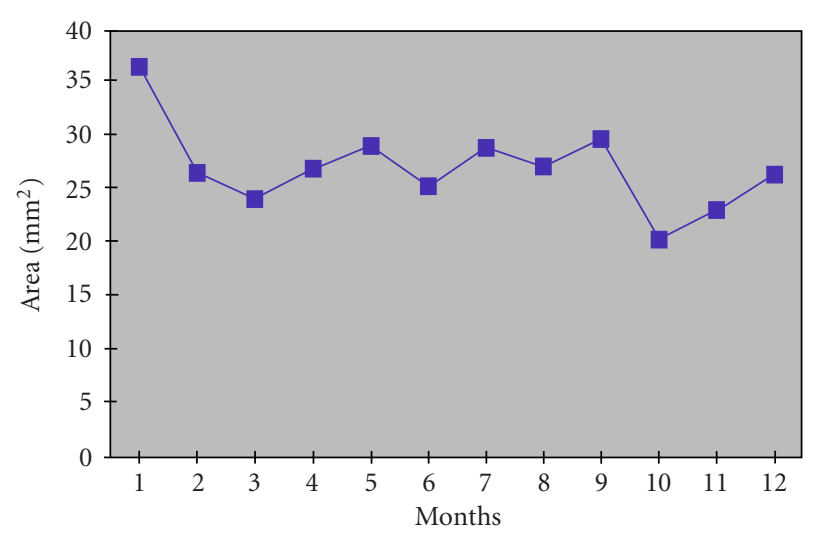

(b)

FIGURE 1. (a) Viability of human neurofibroma xenograft in SCID mouse ear for 12 months after implantation, without therapy with pirfenidone. Human neurofibromas confirmed by frozen section histology were cut in $2 \times 2 \mathrm{~mm}$ pieces and implanted subcutaneously into the ear of SCID mice. Tumor size was assessed by inspection and direct outside measurement of the two largest perpendicular diameters and area calculation, using a caliper with $0.025 \mathrm{~mm}$ accuracy. (b) Neurofibroma xenotransplants in the ear were stable in size for twelve months after implantation. The line shows an average size of four tumor implants in the ears, based on area calculation.

Human S-100 A and B protein was detected using rabbit antiserum (Dako Corp, Carpinteria, Calif) diluted with phosphate-buffered saline (PBS), $\mathrm{pH}=7.2$, containing 5\% bovine serum albumin (BSA), and applied to sections. The rabbit anti-S-100 antibodies were detected using affinity purified biotinylated $\mathrm{F}\left(\mathrm{ab}^{\prime}\right) 2$ swine antirabbit IgG (Dako), followed by horseradish peroxidaseconjugated streptavidin (Dako), both diluted in 5\% PBS/BSA. Specimens were counterstained with alumhematoxylin solution, dehydrated using graded ethanols, cleared in xylene, and coverslipped with a synthetic mounting medium.

Binding of Griffonia Simplicifolia (GS) lectin I, isolectin B4 (IB4), a lectin which binds to Gal $\alpha 1-3 \mathrm{Gal}$ in porcine tissues, was tested in tissue sections prepared as described above [17]. The sections were treated with a microwave-assisted carbohydrate revealing (MCR) by incubating them with $75 \mathrm{~mL}$ of $10 \mathrm{mM}$ citric acid, $\mathrm{pH}=$ 6.0 , and subjecting them to microwave at high power $(800$ watt, Panasonic, Secaucus, NJ) inside a beaker containing $400 \mathrm{~mL}$ distilled water up to 15 minutes. The volume was maintained at a constant level during heating by the addition of distilled water as required. Sections were then incubated for 15 minutes at RT within the residual solution prior to application of the lectin. Fluoresceinconjugated IB4 (Vector Laboratories, Burlingame, Calif) diluted in PBS, $\mathrm{pH}=7.2$, containing 5\% BSA, was applied to the sections at RT for one hour and then rinsed with PBS. Slides were then coverslipped with a $1: 8$ dilution of Vectashield-DAPI $(1.5 \mu \mathrm{g} / \mathrm{mL} 4,6$-diamidino-2phenylindole, Vector Laboratories) in $\mathrm{PBS}, \mathrm{pH}=8.6$, and stored in the dark at $4^{\circ} \mathrm{C}$. Sections from formalin-fixed, paraffin-embedded tissues were also stained with hematoxylin and eosin (H\&E) and Masson's Trichrome [18]. Slides were examined using a brightfield or epifluorescent microscope, and digital photomicrographs were obtained utilizing a high-resolution CCD digital camera (SPOT II, Diagnostic Instruments, Sterling Heights, Mich) mounted to the microscope (Leica DMRD, Leica Microsystems, Inc, Bannockburn, Ill) and SPOT II software.

\section{Statistical methods}

The frequency of surviving tumors was tabulated and compared using the Chi-square test.

\section{RESULTS}

\section{Fate of human neurofibroma xenografts without treatment}

One hundred-six fragments of human neurofibromas were implanted in various locations in 29 SCID mice, and were followed for survival of tumor grafts without therapy for up to six weeks. Two mice with implants under the skin of ear were monitored for twelve months.

The survival of the neurofibroma xenografts in the various locations ranged from $84.6 \%$ to $100 \%$ (Table 1 ). Implants which did not survive could not be found or could not be identified as neurofibromas by inspection. The tissue retrieved in some of these locations appeared pale, soft, and transparent; microscopic evaluation of these samples failed to show characteristic features of neurogenic tumors. Subcutaneously implanted tumors had similar survival rates $(77 / 91)$ as tumors implanted into epineurium of the sciatic nerve $(13 / 15)(P=.37)$. Survival of tumors implanted in the neck and back was similar $(P=.41)$. Implanted tumors showed no aggressive properties or tissue invasion and maintained the same size over the six weeks of the followup, as documented by measurement of tumor mass and volume (data not shown). Neurofibroma xenotransplants in the ear were stable in appearance and size for twelve months postimplantation (Figure 1b). 
TABLE 1. Survival of human neurofibroma xenografts in different locations of SCID mice, without therapy.

\begin{tabular}{lccccccc}
\hline Tumor location & 2 weeks & 3 weeks & 4 weeks & 5 weeks & 6 weeks & 12 months & Total \\
\hline Sciatic nerve & $1 / 2$ & $2 / 2$ & $2 / 3$ & $3 / 3$ & $5 / 5$ & - & $13 / 15(86.7 \%)$ \\
Subcutaneous & $16 / 17$ & $14 / 17$ & $13 / 15$ & $13 / 15$ & $17 / 23$ & $4 / 4$ & $77 / 91(84.6 \%)$ \\
Neck & $9 / 9$ & $7 / 9$ & $7 / 8$ & $7 / 8$ & $11 / 12$ & - & $41 / 46(89.2 \%)$ \\
Back & $7 / 8$ & $7 / 8$ & $6 / 7$ & $6 / 7$ & $7 / 11$ & - & $33 / 41(80.1 \%)$ \\
Ear & - & - & - & - & - & $4 / 4$ & $4 / 4(100 \%)$ \\
\hline Total & & & & & & $90 / 106(84.9 \%)$ \\
\hline
\end{tabular}

TABLE 2. Effect of treatment with pirfenidone for six weeks on survival of human neurofibroma in SCID mice.

\begin{tabular}{l|cc|c}
\hline \multirow{2}{*}{ Survival of xenotransplant } & \multicolumn{2}{|c|}{ Pirfenidone therapy } & \multirow{2}{*}{$P$} \\
\cline { 2 - 3 } & Treated & Not treated & \\
\hline All neurofibromas & $76 / 106(71.7 \%)$ & $86 / 102(84.3 \%)$ & 0.0215 \\
Sciatic nerve & $9 / 15(60.0 \%)$ & $13 / 15(86.7 \%)$ & 0.1077 \\
Subcutaneous & $67 / 91(73.6 \%)$ & $73 / 87(83.9 \%)$ & 0.1361 \\
\hline
\end{tabular}

• Survival of implants was determined by positive histologic identification of neurofibroma. "Failed" implants were defined as an absent tumor or an atypical appearance of retrieved implant without histologic characteristics of neurogenic tumors.

* Four additional tumors implanted in the ear and followed up for 12 months without treatment are not included in this table.

\section{Histologic and immunohistochemical properties of tumors}

Over the 6 weeks of monitoring, human neurofibroma implants retained the histologic characteristics of the original tumors. The presence of collagen within the tumor specimens was confirmed using Masson's trichrome stain (Figure 2). No significant change in cellularity or collagen content was observed, except that tumors appeared more dense in 6-week-old implants (Figures 2 and $3)$. The immunohistochemical reaction with anti-S-100 remained as strong as in the original tumor (Figure 3 ). Vascularization of tumors appeared fully established by histology within seven days and was not changed over six weeks of followup. The blood vessels were of host origin since they bound GS IB 4 , a lectin recognizing Gal $\alpha 1-3 \mathrm{Gal}$ on cells of nonprimates (Figure 4).

Tumors implanted subcutaneously and in the nerve epineurium exhibited minimal histologic differences. Some tumors implanted into nerve epineurium had acute inflammation and some had a granulomatous reaction consisting of histiocytes and multinucleated giant cells in proximity to sutures. Tumors in subcutaneous locations did not have signs of inflammatory response.

\section{EFFECTS OF PIRFENIDONE}

Toxicity and short-term effects of pirfenidone, a novel antifibrotic drug, were explored as a new therapy for NF1. No increased mortality or signs of drug toxicity was observed in animals treated with pirfenidone. Survival of neurofibromas in animals treated with pirfenidone was lower than in control animals, irrespective of the location of implanted tumor (Table 2). Tumor grafts derived from four different patients showed no difference in survival rate. Surviving tumors in treated animals did not show histologic differences from tumors in untreated animals. Tumors in treated animals were vascularized by host blood vessels, similar to tumors in control animals.

\section{DISCUSSION}

Human neurofibromas xenotransplanted into immunodeficient mice offer an attractive experimental model which allows researchers to explore therapies for NF1. Lee et al reported that human neurofibromas transplanted in the sciatic nerve of nude mice grew for over 6 weeks after the implantation, and tumor enlargement and stability correlated with vascularity [16]. The monitoring of changes in tumors implanted in deep tissue, such as subrenal capsule and sciatic nerve epineurium, required surgical exploration of implanted tumors [16]. The assessment of tumor implants in these locations is thus difficult. Further, because deep implants must be secured by sutures, inflammatory responses may ensue, making the monitoring of outcome more difficult. Here we show that human tumors can be implanted in more approachable locations, particularly the ear, where monitoring can be conducted more easily.

The implantation of human tumor under the skin of the ear has the advantage of easy accessibility of the tumor for monitoring of survival, growth, or changes in vascularization. Surgical implantation in this subcutaneous location is rapid and technically straightforward and does not produce substantial trauma nor detectable inflammatory response to surrounding tissues. Our animal model could be used for discovery of new therapies for NF1 


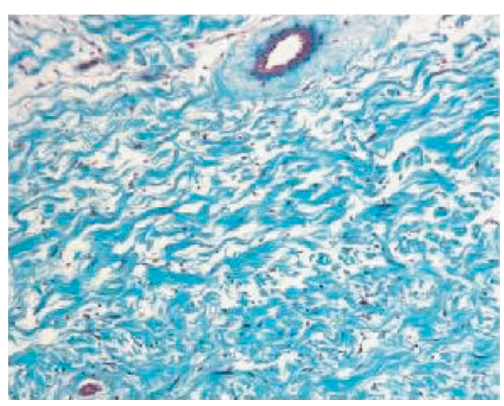

(a)

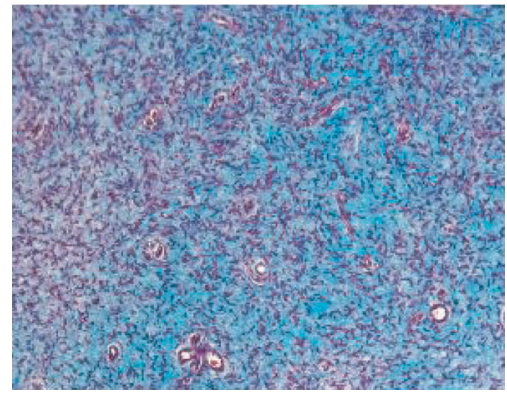

(b)

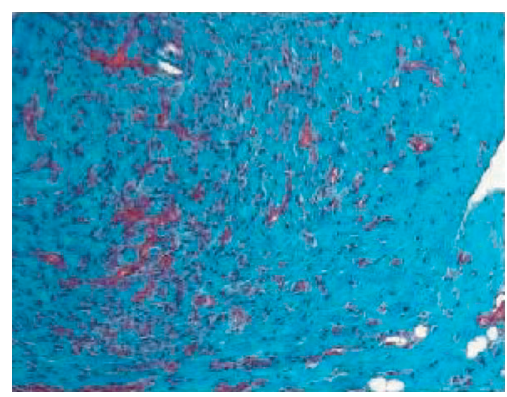

(c)

Figure 2. Evaluation for content of collagen in human neurofibroma xenografts in SCID mouse. Sections from formalinfixed, paraffin-embedded tissues were stained with Masson's Trichrome and slides were examined using a brightfield microscope. Original tumor tissue (a) and the xenotransplant recovered 6 weeks after implantation without therapy (b) or with pirfenidone treatment (c). Samples from the original tumor and 6week-old implants were stained with Trichrome suggesting the presence of similar content of collagen. Tumors recovered from animals treated with pirfenidone did not display difference in Trichrome staining, suggesting that 6-week therapy did not influence the content of collagen.

and might offer an opportunity to study the response of tumors from individuals to various combinations and dosages of therapeutics, allowing customized therapy for NF1.

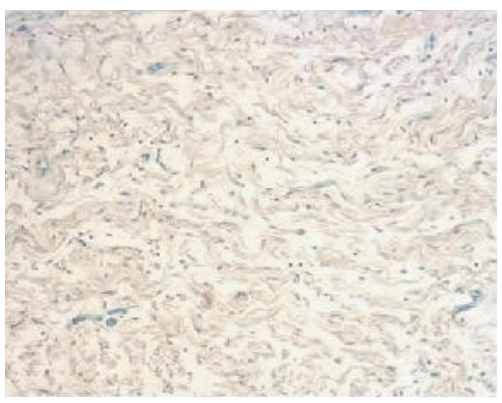

(a)

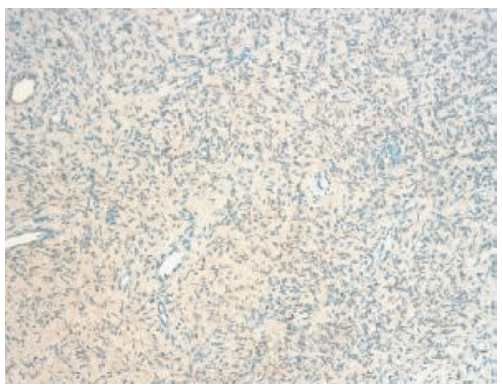

(b)

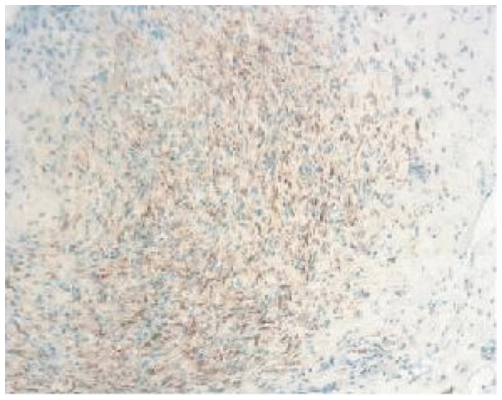

(c)

Figure 3. Localization of S-100 in human neurofibroma transplanted into SCID mouse. Tissue samples from original tumors and from human neurofibroma xenografts were snap-frozen, cryo-sectioned, stained with rabbit antibodies specific for human S-100 and studied by immunofluorescence microscopy. Fluorescence is localized in Schwann cells in the original tumor (a) and in tumor xenografts recovered 6 weeks after implantation from nontreated animal (b) and from animal receiving pirfenidone (c). The presence of S-100 in the xenografts confirms persistence of Schwann cells in implanted tumors. Positivity for S-100 was not changed by treatment with pirfenidone for 6 weeks.

Neurofibromas are benign tumors, with unpredictable pattern of progression. While they may enlarge in early childhood, adolescence, and during the pregnancy, at other times, their growth is mostly indolent. Contrary 


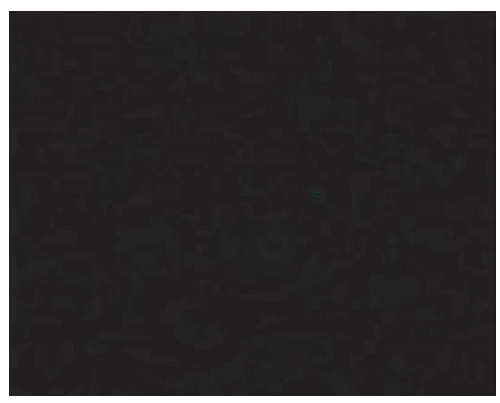

(a)

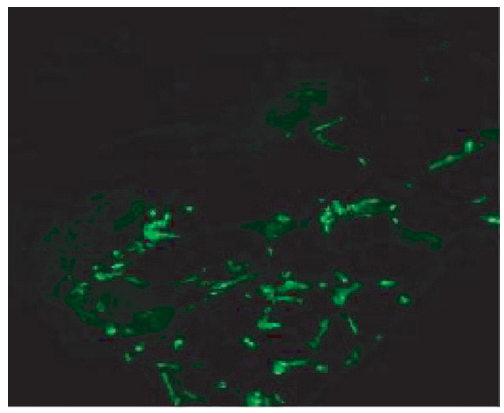

(b)

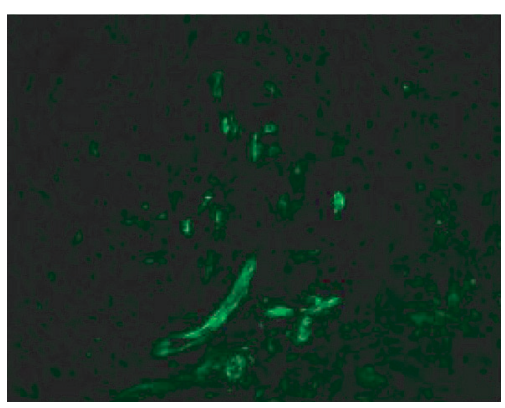

(c)

FIGURE 4. GS IB4 lectin binding in human neurofibroma implanted into SCID mouse. Sections from formalin-fixed, paraffin-embedded tissues from original tumors and from human neurofibroma xenografts were incubated with fluoresceinconjugated IB4. Slides were examined using an epifluorescent microscope. Fluorescence indicates binding of lectin to Gal $\alpha 1-$ $3 \mathrm{Gal}$ on blood vessel cells of nonprimates. Original human tumor (a) does not show fluorescence. Human neurofibroma xenotransplant from nontreated animal (b) and human neurofibroma xenotransplant from animal treated with pirfenidone for 6 weeks (c) show fluorescence suggesting that vessels were of host origin since they bound GS IB4. The host origin of blood vessels was not changed in response to therapy with pirfenidone.

to previous reports [16], we did not observe substantial changes in tumor size after transplant except for modest and transient increase in size in the first week postimplantation. These changes probably reflect tissue swelling as a reaction to the surgical trauma. The lack of measurable growth of tumors in our model did not allow us to evaluate if pirfenidone is capable of arresting tumor enlargement. However, the indolence of xenotransplants probably reflects the general behavior of the tumors in humans. Many factors have been postulated to play a role in the pathogenesis of neurofibromas, including hormones, trauma, growth factors, and surgical manipulations, but a fundamental understanding of events implicated in their development and growth is lacking.

Finally, we have used our experimental model to test the potential toxicity and efficacy of pirfenidone, a new antifibrotic drug, in the treatment of plexiform neurofibromas. Pirfenidone showed no toxicity and reduced survival of neural tumors during the six weeks of study. Our findings do not allow us to suggest by which mechanism(s) pirfenidone inhibits tumor survival. Since the benefit we observed might increase over longer periods of time, additional studies are necessary to evaluate the effects of prolonged therapy with pirfenidone. However, our results indicate that pirfenidone is a good candidate for therapy of neurofibromatosis type I and therefore further investigations are warranted to determine the mechanism of action and long-term effects of treatment with this agent.

\section{ACKNOWLEDGMENTS}

This work was supported by Grants HL52297 and HL46810 (to J.L.Platt), Grant 2A336 from NF, Inc (to D. Babovic-Vuksanovic), and von Liebig Grant 2A3021. We thank Marnac, Inc, for providing the pirfenidone and Ms Lori Adler for assistance in the preparation of this paper. Animal experiments described in this work have been conducted under the protocol approved by the Institutional Animal Care and Use Committee, where national and institutional policies of humane care and use of laboratory animals have been followed (IACUC A30899).

\section{REFERENCES}

[1] Strumf S, Alksne JF, Annegers JF. Neurofibromatosis. Conference statement. National Institutes of Health Concensus Development Conference. Arch Neurol. 1988;45:575-578.

[2] Roos KL, Muckway M. Neurofibromatosis. Dermatol Clin. 1995;13:105-111.

[3] Riccardi VM, Womack JE, Jacks T. Neurofibromatosis and related tumors: natural occurrence and animal models. Am J Pathol. 1994;145:994-1000.

[4] Needle MN, Cnaan A, Dattilo J. Prognostic signs in the surgical management of plexiform neurofibroma - The Children's Hospital of Philadelphia experience 1974-1994. J Pediatr. 1997;131:678-682.

[5] Packer RJ, Gutmann DH, Rubenstein A, et al. Plexiform neurofibromas in NF1: toward biologic-based therapy. Neurology. 2002;58(10):1461-1470. 
[6] Sheela S, Riccardi VM, Ratner N. Angiogenic and invasive properties of neurofibroma Schwann cells. $J$ Cell Biol. 1990;111(2):645-653.

[7] Appenzeller O, Kornfeld M, Atkinson R, Snyder RD. Neurofibromatosis xenografts. Contribution to pathogenesis. J Neurol Sci. 1986;74(1):69-77.

[8] Muir D, Neubauer D, Lim IT, Yachnis AT, Wallace MR. Tumorigenic properties of neurofibromindeficient neurofibroma Schwann cells. Am J Pathol. 2001;158(2):501-513.

[9] Kaneko M, Inoue H, Nakazawa R, et al. Pirfenidone induces intercellular adhesion molecula-1 (ICAM1) down-regulation on cultured human synovial fibroblasts, Clin Exp Immunol. 1998;113:72-76.

[10] Zhang A, Shiels IA, Ambler JS, Taylor SM. Pirfenidone reduces fibronectin synthesis by cultures human retinal pigment epithelial cells. Aust $N Z J$ Ophthalmol. 1998;26(suppl 1):S74-S76.

[11] Shimizu T, Kuroda T, Hata S, Fukagawa M, Margolin SB, Kurokawa K. Pirfenidone improves renal function and fibrosis in post-obstructed kidney. Kidney Int. 1998;54:99-109.

[12] Iyer SN, Gurujeyalakshmi G, Giri SN. Effects of Pirfenidone on procollagen gene expression at the transcriptional level in bleomycin hamster model of lung fibrosis. J Pharmacol Exp Ther. 1998;189:211-218.

[13] Peltonen J, Marttala T, Vihersaari T, Renvall S, Penttinen R. Collagen synthesis in cells cultured from v. Recklinghausen's neurofibromatosis. Acta Neuropathol (Berl). 1981;55(3):183-187.

[14] Uitto J, Matsuoka LY, Chu ML, Pihlajaniemi T, Prockop DJ. Connective tissue biochemistry of neurofibromas. Ann N Y Acad Sci. 1986;486:271-286.

[15] Bosma MJ, Carroll AM. The SCID mouse mutant: definition, characterization, and potential uses. Annu Rev Immunol. 1991;9:232-250.

[16] Lee JK, Sobel RA, Chiocca EA, Kim TS, Martuza RL. Growth of human acoustic neuromas, neurofibromas and schwannomas in the subrenal capsule and sciatic nerve of the nude mouse. J Neurooncol. 1992;14:101-112.

[17] Mattsson G, Carlsson PO, Olausson K, Jansson L. Histological markers for endothelial cells in endogenous and transplanted rodent pancreatic islets. Pancreatology. 2002;2(2):155-162.

[18] Masson P. Trichrome stainings and their preliminary technique. J Tech Meth. 1929;12:75-90.

\footnotetext{
* Corresponding author.

E-mail: dbabovic@mayo.edu

Fax: +1 507285 1067; Tel: +1 5072843215
} 


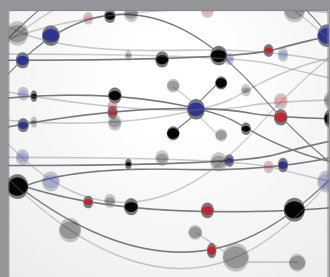

The Scientific World Journal
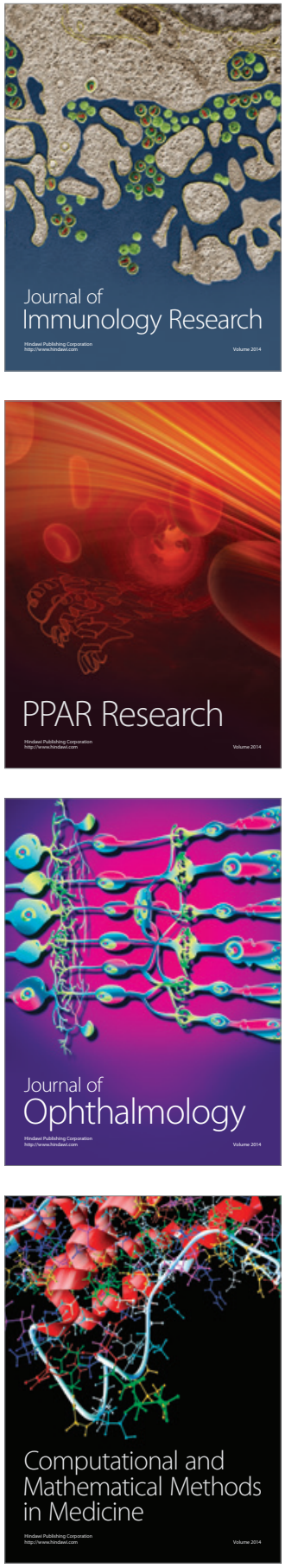

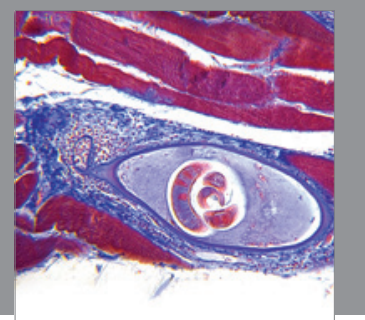

Gastroenterology

Research and Practice
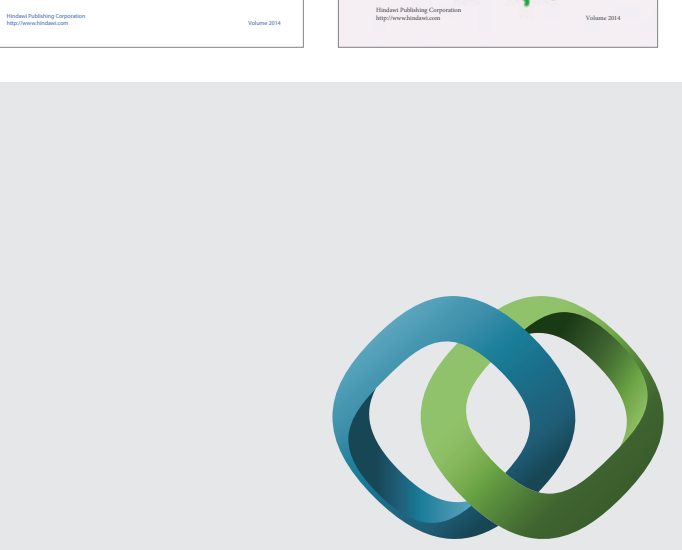

\section{Hindawi}

Submit your manuscripts at

http://www.hindawi.com
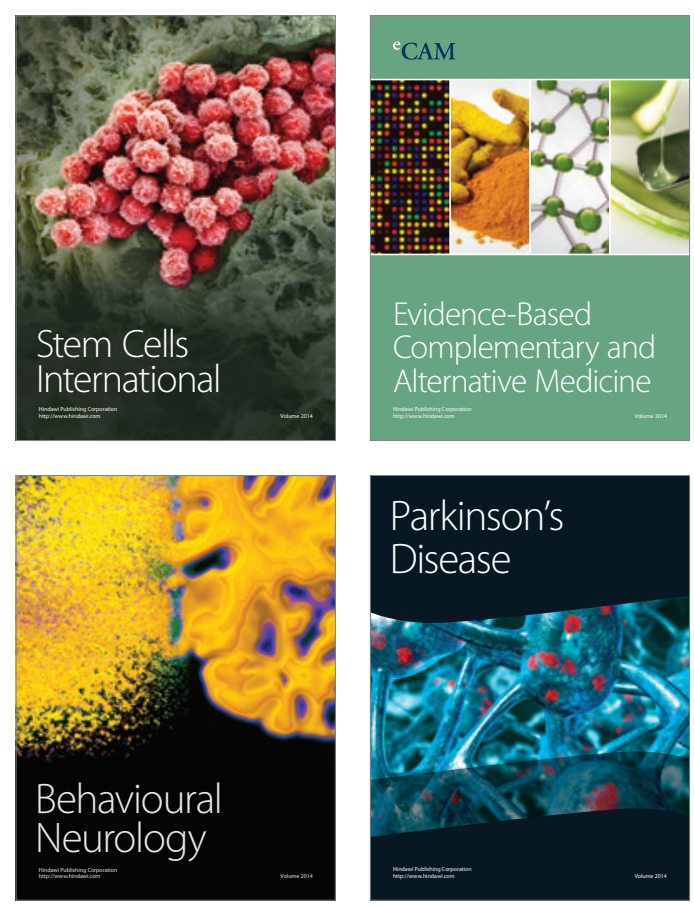

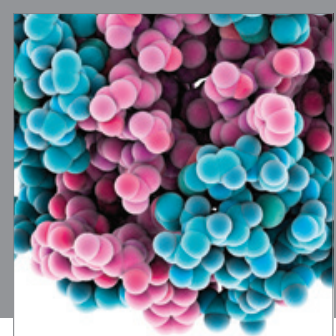

Journal of
Diabetes Research

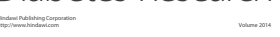

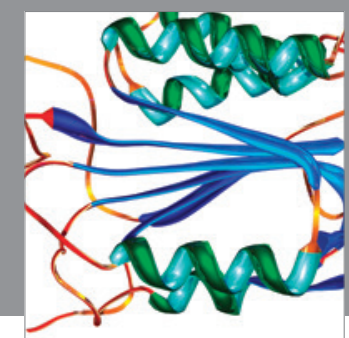

Disease Markers
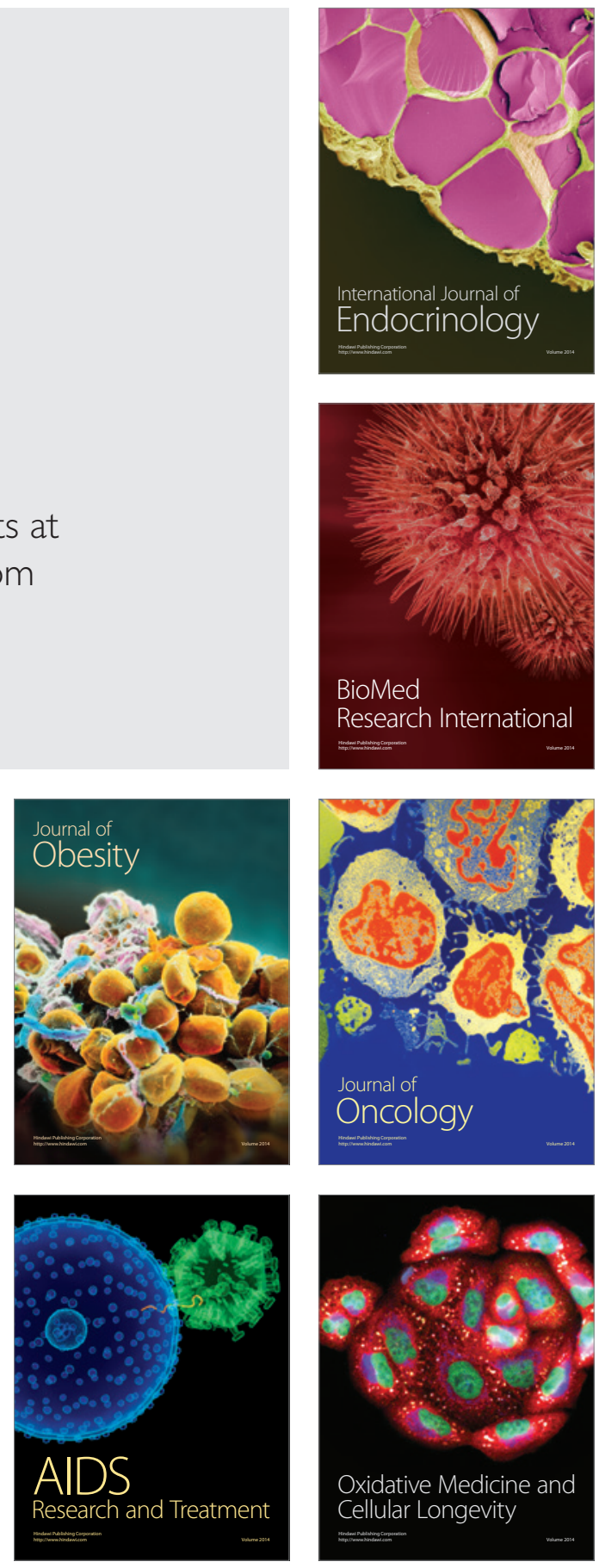